\title{
Engineering Student Perspectives on Research and What It Means to Be a Researcher
}

\section{Dr. Lisa Benson, Clemson University}

Lisa Benson is a Professor of Engineering and Science Education at Clemson University, with a joint appointment in Bioengineering. Her research focuses on the interactions between student motivation and their learning experiences. Her projects involve the study of student perceptions, beliefs and attitudes towards becoming engineers and scientists, and their problem solving processes. Other projects in the Benson group include effects of student-centered active learning, self-regulated learning, and incorporating engineering into secondary science and mathematics classrooms. Her education includes a B.S. in Bioengineering from the University of Vermont, and M.S. and Ph.D. in Bioengineering from Clemson University.

\section{Dr. Courtney June Faber, University of Tennessee, Knoxville}

Courtney is a Research Assistant Professor and Lecturer in the Cook Grand Challenge Engineering Honors Program at the University of Tennessee. She completed her Ph.D. in Engineering \& Science Education at Clemson University. Prior to her Ph.D. work, she received her B.S. in Bioengineering at Clemson University and her M.S. in Biomedical Engineering at Cornell University. Courtney's research interests include epistemic cognition in the context of problem solving, and researcher identity.

\section{Dr. Rachel Louis Kajfez, Ohio State University}

Dr. Rachel Louis Kajfez is an Assistant Professor in the Department of Engineering Education at The Ohio State University. She earned her B.S. and M.S. degrees in Civil Engineering from Ohio State and earned her Ph.D. in Engineering Education from Virginia Tech. Her research interests focus on the intersection between motivation and identity of undergraduate and graduate students, first-year engineering programs, mixed methods research, and innovative approaches to teaching.

\section{Dr. Marian S. Kennedy, Clemson University}

Marian Kennedy is an Associate Professor within the Department of Materials Science \& Engineering at Clemson University. Her research group focused on the mechanical and tribological characterization of thin films. She also contributes to the engineering education community through research related to undergraduate research programs and navigational capital needed for graduate school.

\section{Ms. Katherine M. Ehlert, Clemson University}

Katherine M. Ehlert is a doctoral student in the Engineering and Science Education department in the College of Engineering, Computing, and Applied Sciences at Clemson University. She earned her BS in Mechanical Engineering from Case Western Reserve University and her MS in Mechanical Engineering focusing on Biomechanics from Cornell University. Prior to her enrollment at Clemson, Katherine worked as a Biomedical Engineering consultant in Philadelphia, PA. Her research interests include identity development through research experiences for engineering students, student pathways to engineering degree completion, and documenting the influence of co-op experiences on academic performance.

\section{Dennis M. Lee, Clemson University}

Dennis M. Lee is a doctoral student in the Engineering and Science Education Department and Graduate Research Assistant in the office of the Associate Dean for Undergraduate Studies in the College of Engineering, Computing, and Applied Sciences at Clemson University. He received his BA and MS in bacteriology from the University of Wisconsin, Madison. Prior to his studies at Clemson University, he taught introductory biology at Tri-County Technical College in Pendleton, SC. His research interests include the development of researcher identity and epistemic cognition in undergraduate STEM students.

\section{Ms. Anne Marguerite McAlister, Ohio State University}




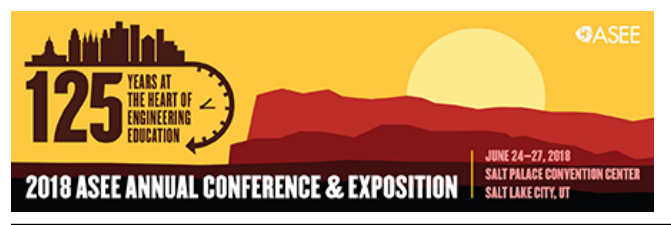

Paper ID \#23194

Anne is an undergraduate student at The Ohio State University studying chemical engineering.

\section{Teresa Porter, Ohio State University}




\title{
Engineering Student Perspectives on \\ Research and What It Means \\ to Be a Researcher
}

\begin{abstract}
Introduction
Undergraduate research experiences (UREs) have shown positive effects on students' understanding of the nature of science, motivation, and academic performance. However, little is understood about how UREs affect students' views of research and being researchers. The importance of understanding students' identities as researchers lies in the alignment of their research skills with key aspects of their epistemic beliefs, or ways of knowing fundamental concepts in engineering and how to practice engineering. Our overarching research question is: How do undergraduate engineering students develop their identities as researchers and their ways of knowing engineering through research experiences? The outcomes of understanding how undergraduates develop researcher identities and engineering epistemic beliefs will inform the development of engineering education experiences to provide meaningful ways for students to engage, function, and learn in both traditional research and research-infused classroom learning environments.
\end{abstract}

The research questions being explored in this project are:

1. How do undergraduate engineering students conceptualize and construct what it means to be a researcher?

2. What do these students perceive to be the factors that affect their researcher identity development?

3. How do these students conceptualize and interpret epistemic frameworks of their fields (i.e. the nature of knowledge and knowing)?

4. How do beliefs about the nature of knowledge and knowing develop within the contexts of students' research experiences?

We are conducting a multi-institution, multi-phase, mixed methods project using a grounded theory approach to address our research questions and expand existing identity and epistemic belief theories. In Phase I of the project, a survey with both close-ended and open-ended questions was used to gather data on research questions 1, 2, and 3 from engineering students from 4 institutions. Results informed interview questions and participant selection in Phase II, which entailed semistructured interviews to explore relationships between students' epistemic beliefs and identity in the context of research. Quantitative and qualitative data from Phases I and II are being mixed throughout, from data collection and handling to analysis and interpretation ${ }^{1}$. Themes emerging from the interviews will inform an engineering-specific learning theory based on students' epistemic beliefs and researcher identities as they engage in engineering research to address research question 4. This theory will be applied in Phase III to inform engineering educators and develop ways to integrate findings into engineering degree programs. Our target population for all phases and data types includes Mechanical Engineering (ME) and Biomedical Engineering/Bioengineering (BME) undergraduate students who have participated in research in some capacity. 


\section{Summary of findings}

1) Evidence-based interview protocol about relationships between epistemic cognition and identity in the context of research

Results from our pilot study and Phase I survey data analysis ${ }^{2}$ revealed four themes based on factors that students perceived as affecting their researcher identities: discovery, dissemination, integration into society, and self-regulation. These themes were used to develop a semi-structured interview protocol that included base questions and follow-up questions; follow-up questions allowed us to explore topics that participants were not initially able to verbalize on their own. For example, one base question asked about where knowledge in the field comes from; a follow-up question was added to prompt participants to identify ways they can produce knowledge in their field. This thread provided the opportunity to find links between identity and epistemic thinking.

Following a grounded theory approach, our interview protocol has evolved in response to ongoing analysis of interview data. For example, when evidence of epistemic thinking was emerging, we added more follow-up questions in subsequent interviews to explore specific aspects of epistemic thinking such as epistemic cognition and epistemic metacognitive skill, knowledge and experience.

To date, 22 interviews have been completed, and 14 interviews have been analyzed. Themes emerging from those interviews are showing that many of our participants conveyed sophisticated but differing definitions of what it means to be a researcher, and their perceptions of researchers positively and negatively influenced students’ researcher identity.

2) Use of structured memoing to support a grounded theory approach and mixed-methods analyses

The general process for coding interview data, which is currently ongoing, is to initially apply theory-level codes related to identity and epistemic thinking, followed by more specific codes for student beliefs, attitudes, and practices.

Theory-level codes were developed to expand codes beyond those created from open-ended survey data, which did not fully align with data emerging from interviews of the participants. The theorylevel codes were used to isolate salient passages without the restriction of a more detailed codebook. Two main theories were used to develop theory-level codes:

- Epistemic ${ }^{3,4}$ : Epistemic cognition; epistemic metacognition; epistemic metacognitive skills, knowledge and experience

- Identity ${ }^{5,6}$ : Participants’ researcher identities and perceptions of researchers; Nature Identity; Affinity Identity; Preferential Identity; Institutional Identity; Discourse (Person) Identity; Discourse (Material) Identity

These theory-level codes are important as we build our grounded theory; they define what the theory should be regarding the interconnections between epistemic thinking and identity.

Specific codes were generated from in vivo coding of Phase I open-ended survey responses about what students describe that they are doing as part of their research experiences. From this list of potential codes, codes were identified based on what was found in Phase II interview transcripts. These include activities such as constructing knowledge that is new to participant or 
new to field, collaboration, testing ideas, and dissemination. Some codes reflect students' attitudes and beliefs, such as career goals or plans; challenges they faced when doing research; and aspects of mentoring or supervision. Other codes reflect aspects of students' experiences, such as recognition, failure, gaining skills, or feeling a sense of gratification. These codes will be particularly important for Phase III of this project, in which we will identify ways to transfer our findings to instructional practice and more traditional educational settings such as classrooms and labs. These codes reflect how students perceive the research process.

For each transcript, two coders reviewed the transcript independently (blinded to each other's code applications and reflections) using Dedoose, an online mixed-methods analysis software ${ }^{7}$, applying theory-level codes for epistemic thinking and identity, and specific codes for students' descriptions of their attitudes, beliefs and perceptions about research processes. The two coders reflected on each coded passage using reflections connected to the applied code(s) to explain why they applied the code or combination of codes. In cases where multiple codes were applied to the same passage, reflections included a discussion on how these theory-level codes interacted with each other in the passage. Once the transcript was coded, a structured memo was co-constructed by the coders including a description of the participant, meaning-making about the participants' perceptions of research, researcher identity and ways of knowing in engineering and research. Memoing is key to the process of theoretical coding to generate categories, form hypotheses about the relationships between epistemic thinking and researcher identity, and to test hypotheses about these relationships. For example, do certain kinds of identity co-vary with certain kinds of epistemic thinking? With respect to specific codes, do certain experiences or contexts influence identity and epistemic thinking? As our grounded theory study progresses, we have multiple options for testing hypotheses. We can modify our interview protocol to ask about hypothesized relationships (similar to member checking) for the last round of interviews, review memos to look for evidence of these relationships, and conduct mixed methods data analysis as the quantitative data allows to test hypotheses within quantitative survey data.

\section{3) Use of quantitative survey items as an interview prompt}

As part of both the survey and interview, participants were asked to rate how much they feel like a researcher using a scale of 1 (No, not at all) to 7 (Yes, very much). During the interviews, participants were asked why they picked their rating. Our analysis of interview data revealed that participants interpreted the end-points of the scale differently, their ratings changed between the time they completed the survey and the interview, because their definitions of research changed during that time, and some ratings decreased even though the participants believed they were better researchers. Using the numerical scale within the interview allowed for the participants to elaborate on their views of what it means to be a researcher. Using follow-up questions, the interviewers were able to often connect a participant's researcher identity with their epistemic views. The interview data enhanced our interpretation of participants' survey ratings, allowing us to capture richer data and align our interpretation through the participant's perspective.

\section{Conclusions}

Although we have not begun Phase III of this project, in which we translate our research findings to educational practice, we can identify aspects of undergraduate research experiences that can build positive perceptions of research, researcher identity, and epistemic thinking, both for UREs and classroom practices. From our initial findings, these practices include: 
- UREs:

o Allow students to observe and reflect on research practices and processes prior to participating on a project.

o Refer to students as researchers, both in correspondence and in face-to-face meetings

o Explicitly identify for students which activities are part of research process

- Classroom environments:

o Have students reflect on how knowledge in their field has changed over time and discuss the process of creating new knowledge.

o Plan and conduct open-ended laboratory experiences or solve problems for which students and instructors do not know the outcome.

o Ask students to reflect on the knowledge they gained that is new to them versus new to their field.

\section{Future Work}

The outcome of the first two phases of this work will be the development of a theoretical model that captures epistemic cognition and identity development during UREs based on our data and using a grounded theory approach. The final phase of the project will involve defining and describing the factors and experiences from UREs that students identified as having contributed to their researcher identity and transformed their epistemic beliefs. These factors and experiences will be translated to educational learning environments to leverage findings such that students in more traditional learning environments can benefit in the same ways as students who participate in UREs, such as developing identities as builders of new knowledge, as contributing to society and as effective communicators.

\section{Acknowledgements}

This research was funded through a grant from the National Science Foundation (Award \# 1531607 and 1531641).

\section{References}

[1] Benson, L., Faber, C. J., Kajfez, R., Ehlert, K., Lee, D. \& McAlister, M. Assessing Undergraduate Engineering Students' Researcher Identity and Epistemic Cognition. Proceedings of the 124th American Society for Engineering Education Annual (ASEE) Conference \& Exposition, Columbus, OH. (June 25 - 28, 2017).

[2] Kajfez, R., McAlister, A. M., Faber, C. J., Ehlert, K. M., Lee, D. M., Benson, L. C., \& Kennedy, M. S. Exploring Undergraduate Engineering Researchers' Identities and Epistemologies: Who Are They and What Do They Believe? American Education Research Association (AERA) Annual Conference, San Antonio, TX, April 2017.

[3] Chinn, C. A., Rinehart, R. W., \& Buckland, L. A. (2014). Epistemic cognition and evaluating information: Applying the AIR model of epistemic cognition. In D. Rapp \& J. Braasch (Eds.), Processing Inaccurate Information: Theoretical and Applied Perspectives from Cognitive Science and the Educational Sciences (pp. 425-453). Cambridge, MA: EBSCO Publishing. 
[4] Barzilai, S. \& Zohar, A. (2016) Epistemic (Meta) cognition: Ways of thinking about knowledge and knowing. In J. Greene, W. Sandoval, \& I. Braten (Eds.), Handbook of Epistemic Cognition (pp. 409—424). New York, NY: Routledge.

[5] Gee, J. P. (2001). Identity as an Analytic Lens for Research in Education. Review of Research in Education, 25, 99-125.

[6] Weiner, S., Lande, M., \& Jordan, S. S. (2017). Making identities: Understanding the factors that lead young adults to identify with the maker movement. In American Society for Engineering Education Annual Conference (June).

[7] Dedoose Version 8.0.31, web application for managing, analyzing, and presenting qualitative and mixed method research data (2018). Los Angeles, CA: SocioCultural Research Consultants, LLC www.dedoose.com. 